\title{
Anagram solution times as a function of initial visual pattern: Familiar vs unfamiliar typeface*
}

\author{
CHRISTOPHER PETERSON \\ University of Colorado, Boulder, Colo. 80302
}

\begin{abstract}
Sixteen undergraduates each solved eight anagrams. The typeface in which the anagrams were presented was varied between Ss, and letter moves until solution were varied within Ss. Problems presented in a familiar typeface were more quickly solved than problems presented in an unfamiliar typeface $(p<.005)$. This effect was more pronounced for 3 -move problems than for 1 -move problems $(p<.05)$. It was concluded that anagram solution involves a covert rearrangement of the anagram's letters, and that this rearrangement has an imaginal element under partial control of the initial visual pattern of the anagram.
\end{abstract}

The bulk of the anagram research has been concerned with the investigation of the verbal parameters of anagram solution. The effects of word frequency, transition probabilities, and letter order have been well studied, justifying the comment that "we know more about the anagram problem than any other [Johnson, 1966; p. 371]." Because the verbal parameters of the anagram task are fairly well known, it is possible to investigate the less obvious components of anagram solution. The investigation of the initial visual pattern of the anagram seems to be a potentially fertile direction for anagram research to follow. One possibility allowed by such investigation is a refined conceptualization of the implicit processes involved in anagram solution. Most theoreticians (e.g.. Hunter, 1959; Mayzner, Tresselt, \& Helbock, 1964; Underwood, 1966; Johnson, 1966) posit a covert rearrangement of the anagram's letters as part of the solution process. A question not answered regards the nature of the rearrangement. Is it a solely verbal encoding that is covertly rearranged, or is there an imaginal component of the encoding that is under partial control of the initial visual pattern of the anagram?

The present study investigated the latter possibility. If the covert encoding that takes place during anagram solution in under partial control of the initial visual pattern of the anagram, then it should be possible to manipulate certain characteristics of this encoding in a way that differentially affects solution times by manipulating analogous properties of the anagram's visual pattern. On the other hand, if the covert encoding is solely verbal, then manipulation of these visual parameters should not differentially affect solution times.

The manipulation employed in the present study was the familiarity of the typeface in which the anagram problem was presented. If the covert rearrangement of the anagram's letters is under partial control of the

\footnotetext{
*Sponsored by Bruce Ekstrand, who takes full editorial responsibility for its contents.
}

typeface, then an unfamiliar typeface should lead to an unfamiliar encoding. Presumably, an unfamiliar encoding should be more difficult to manipulate than a familiar one and should result in longer solution times.

It was realized that differences in solution times might occur as a function of typeface merely because of input differences and not because of covert manipulability differences. For this reason, the number of letter moves until anagram solution occurred was also manipulated. Letter moves can be interpreted in terms of the amount of covert manipulation required for solution (Dominowski, 1966). A 3-move anagram requires more covert rearranging than a 1 -move anagram. For the given typeface, no perceptual differences should exist between 1 -move and 3-move anagrams. If the retarding effect of an unfamiliar typeface on anagram solution times is constant across letter moves, then no explanation beyond input differences is warranted. However, if this retarding effect is more pronounced for 3-move anagrams than for 1-move anagrams, then a purely perceptual explanation is untenable, and support is given to the notion that imaginal processes play a part in anagram solution.

If the covert rearrangement of an anagram's letters during solution has an imaginal element under partial control of the familiarity of the typeface in which the anagram is presented, then (a) due to both perceptual differences and manipulability differences, problems presented in a familiar typeface should be easier to solve than problems set in an unfamiliar typeface, and (b) due to manipulability differences, the differences between problems presented in a familiar typeface and those presented in an unfamiliar typeface should be more pronounced for 3-move solutions than for 1-move solutions.

\section{METHOD \\ Subjects}

Sixteen undergraduates at the University of Colorado, fulfilling a requirement for introductory psychology, served as Ss in this experiment. Ss were assigned randomly to two groups.

\section{Design}

A 2 by 2 factorial design was employed, with typeface (familiar or unfamiliar) as a between-Ss factor and letter moves (1 or 3 ) as a within-Ss factor. Each $\mathrm{S}$ solved anagrams of the same eight words with word order and letter order varied in a $8 \mathrm{x}$ $8 \mathrm{Greco}-\mathrm{Latin}$ square, so that each word was solved once in each serial position and once with each letter order. The same square was used for both groups.

\section{Words}

Eight solution words (banjo, basic, covet, incur. lanky, limbo, mince, olden) were chosen randomly from the Thorndike \& Lorge (1944) tables, subject to the following restrictions: frequency. $1-10$ per million. 5 letters $(3$ consonants and ? 
vowels): no repeated letters: no proper nouns: no contractions: single solution.

\section{Anagrams}

Dominowski's (1966) letter orders were used. Four 1 -move solution orders $(12435,23145,31245,51234)$ and four 3 -move solution orders (15432. 35241.43521.53421) were randomly chosen from those he presented.

\section{Stimulus Material}

The anagrams were centered on $3 \times 5$ white index cards. Typeface was varied using commercially available transfer letters The familiar typeface was $1 / 4$-in. black Gothic (Stenso No. 20-303), and the unfamiliar typeface was $1 / 4$-in. black Old English (Quik Stik No. 745). Letter spacing was identical for both typefaces.

\section{Procedure}

Ss were run individually. Instructions were given to each $\mathrm{S}$ and a sample anagram was solved. The $S$ then attempted to solve the eight anagrams. They were presented sequentially, and up to 4 min was allowed for each solution. To make sure that all Ss perceived the letters correctly, the E pronounced, in order, the letters in the anagram as he first presented it to the $S$. If solution did not occur in the 4-min period. a time of $4 \mathrm{~min}$ was recorded and the correct solution was given. Time until solution was recorded to the nearest 10 th of a second.

\section{RESULTS}

Because solution times were skewed. medians for each $\mathrm{S}$ were used in the analysis. The data from one $\mathrm{S}$ in the familiar typeface condition were replaced because he failed to solve six of the eight problems. All the other Ss solved at least half the problems. The means of the medians in the different conditions were (1) familiar 1-move: $17.1 \mathrm{sec}$. (2) unfamiliar 1-move: $39.8 \mathrm{sec}$. (3) familiar 3 -move: $67.5 \mathrm{sec}$, (4) unfamiliar 3-move: $149.7 \mathrm{sec}$.

A $2 \times 2$ analysis of variance was computed, with typeface as a between-Ss factor and letter moves as a within-Ss factor. The results supported the hypotheses of the experiment. Problems presented in a familiar typeface were more quickly solved than problems presented in an unfamiliar typeface. $F(1,14)=13.4120$. $\mathrm{p}<.005$. Problems with 1 -move solutions were more quickly solved than problems with 3 -move solutions, $F(1,14)=36.6138, p<.001$. The difference in solution times between familiar typeface problems and unfamiliar typeface problems was greater for 3-move solutions than for 1 -move solutions, $F(1,14)=5.0232, p<.05$.

\section{DISCUSSION}

The results of the experiment are clear. Anagram problems presented in a familiar typeface are more quickly solved than problems presented in an unfamiliar typeface, and this difference becomes more pronounced as the number of letter moves until solution occurs is increased. These results can be interpreted as supporting the hypothesis that anagram solution involves a covert encoding of the anagram's letters under partial control of the anagram's initial visual pattern. Ss' subjective reports informally supported this interpretation. It was frequently remarked by $\mathrm{Ss}$ in the unfamiliar typeface condition that the letters were difficult to visualize and that the solutions were difficult to recognize.

\section{REFERENCES}

Dominowski, R. L. Anagram solving as a function of lette moves. Journal of Verbal Learning \& Verbal Behavior, 1966 , 5, 107-111.

Hunter, I. M. L. The solving of five-letter anagram problems. British Journal of Psychology, 1959, 50, 193-206.

Johnson, D. M. Solution of anagrams. Psychological Bulletin $1966,66,371-385$.

Mayzner, M. S., Tresselt, M. E., \& Helbock, H. An exploratory study of mediational responses in anagram problem solving. Journal of Psychology, 1964, 57, 263-274.

Thorndike, E. L., \& Lorge, I. The teacher's word book of 30,000 words. New York: Teachers College, Columbia University, 1944.

Underwood, B. J. Experimental psychology. New York: Appleton-Century-Crofts, 1966.

(Received for publication October 9, 1973.) 